\title{
Kowakare: A New Perspective on the Development of Early Mother-Offspring Relationship
}

\author{
Koichi Negayama
}

Published online: 15 December 2010

(C) The Author(s) 2010. This article is published with open access at Springerlink.com

\begin{abstract}
The mother-offspring relationship has components of both positivity and negativity. Kowakare is a new concept introduced to explain an adaptive function of the negativity in the early mother-offspring relationship. Kowakare is the psychosomatic development of the relationship as the process of accumulation in the otherness of offspring. Early human Kowakare has two frameworks, biological interbody antagonism and socio-cultural allomothering compensating the antagonism. Some features of feeding/weaning, parental aversion to offspring's bodily products, and transition from dyad to triad relationship (proto-triad relationship) in tactile play are discussed. Early human Kowakare is promoted by allomothering with the nested systems of objects/persons/institutions as interfaces between mother and offspring. Kowakare makes mother-offspring relationship a mutually autonomous and cooperative companionship.
\end{abstract}

Keywords Kowakare · Mother-offspring relationship · Otherness · Weaning · Prototriad · Autonomy

\section{What is Kowakare?: Mechanism Underlying Bio-social Development of 'Otherness'}

The ultimate goal of child-care is a state of mutual autonomy and companionship between mother and offspring. The development of the mother-offspring relationship is a process of parting between them. 'Kowakare' is a Japanese term often used to describe offspring independence triggered by the mother's aggression directed to the offspring among the Carnivores. It is adopted here to explain the adaptive antagonism and parting between mother and offspring in both animals and humans. It is a psycho-somatic or bio-social mechanism working to promote mutual

\footnotetext{
K. Negayama ( $\square)$

Department of Human Environmental Sciences, Faculty of Human Sciences,

Waseda University, 2-579-15 Mikajima, Tokorozawa, Saitama 359-1192, Japan

e-mail: negayama@waseda.jp
} 
autonomy in mother-offspring relationship. It begins at the prenatal period and ends with the death of either mother or offspring. It is underpinned by conflict between mother and offspring, and includes both aggression/rejection and leaving/departure.

Kowakare in early development can be expressed as a process of accumulation in the offspring's various bio-social 'otherness' for the mother. The offspring is immunologically an alien which ought to be rejected from the beginning of life (immunological other), and mammals are unique in their system of immunological tolerance to a fetus to be kept in the mothers' uterus. Parturition is the end of the intrauterine protection, and therefore is a separation from the mother, not an encounter with her. The neonate then actively interacts with the mother, by sucking for instance (behavioral other). Holding is a way to protect young offspring after parturition, and crawling and walking mean departure from the protective mother. Weaning is a cessation of the offspring's nutritional dependence on the mothers' milk (nutritional other), which has been discussed as a typical mother-offspring conflict (Trivers 1974). Children are soon enrolled in day nurseries, kindergartens or schools, and their activity range rapidly extends outside the home (social other).

Attachment (Bowlby 1969) is a fundamental behavioral system of offspring which seeks the security of protection by the maternal figure through the establishment of a mutual intimate relationship. Bowlby's idea is related to Harlow (1958)'s well-known experiment with surrogate mothers accepting contact-seeking rhesus monkey infants (Van der Horst et al. 2008).

It is misleading, however, to focus exclusively on the positive side of the motheroffspring relationship. It is just one side of a coin, and the relation has a negative aspect as well. The surrogate mother in Harlow's experiment never rejects the offspring. Rosenblum and Harlow (1963) conducted an experiment in which a burst of compressed air was delivered from the surrogate that mimicked rejection by mother. The result showed that it was not effective in promoting independence of the offspring. However, the experiment is flawed as the negative stimulus in the experiment was inflicted irrespective of the infant's behavior, which would be a case of non-contingent negativity at an inappropriate time.

Negative behavior of mothers has been regarded as a precursor of children's later aggression in the context of attachment theory (Main and Stadtman 1981), but sociobiology gave an alternative viewpoint to offspring-directed negative behavior: It is interpreted as being adaptive within a framework of weaning conflict based on the costs and benefits of nursing the infant in terms of parental investment (Trivers 1974). In other words, sociobiology views it as the mother's reproductive strategy to leave the developing offspring behind and promote its autonomy, and then to regain her reproductive status. What is important here is the fact that care of offspring could be a repetitive process rather than a singular experience in the female.

The mother-offspring relationship could be regarded as an instance of the 'porcupine's dilemma' with ambivalence between protection/dependency and rejection/resistance. Adding the aspect of negativity to the discussion of the mother-offspring relationship widens our understanding of the communication between them, and also allows us to have a more dynamic view of motheroffspring interactions. It gives us a more holistic understanding of the relationship. In other words, the framework of Kowakare is complementary with that of attachment. 
Japanese monkey mothers normally show aggression to their offspring at a certain period in the course of the offspring's development caused by irritability (Negayama 1981). The aggression has the positive function of promoting independence in the offspring, and of emancipating the mother from the burden of care as well. The mother-offspring relationship of vervet monkeys consists of both positive and negative components (Fairbanks and McGuire 1988). Negative behavior by rhesus monkey mothers is related to the earlier conception and hence earlier parturition of the next infant (Simpson et al. 1981). Hansen (1966) pointed out the existence of negative maternal behavior in rhesus monkeys under a category of 'punishment'. This is a term used in the domain of learning when functioning to reduce an undesirable behavior, and the interpretation may be different from mine as aggression arising from the mothers' irritation.

Negative interactions between mother and offspring have an adaptive function for the development of their mutual autonomy. What is important is the fact that the effectiveness of the negativity depends much on its timing and intensity. If it appears at a proper moment with a proper intensity, the offspring will increase in autonomy rather than in dependency. This idea is similar to Separation-Individuation theory (Mahler and Perriere 1965), but my framework is more biological and bodyoriented.

Cross-species comparison of mother-offspring relationships in the first year of life among 14 primate species in a caged condition and two human groups (from Japan and the UK), revealed three major different types of parting (Fig. 1; Negayama 1996, 2002): (1) quick parting without antagonism in which the offspring take the

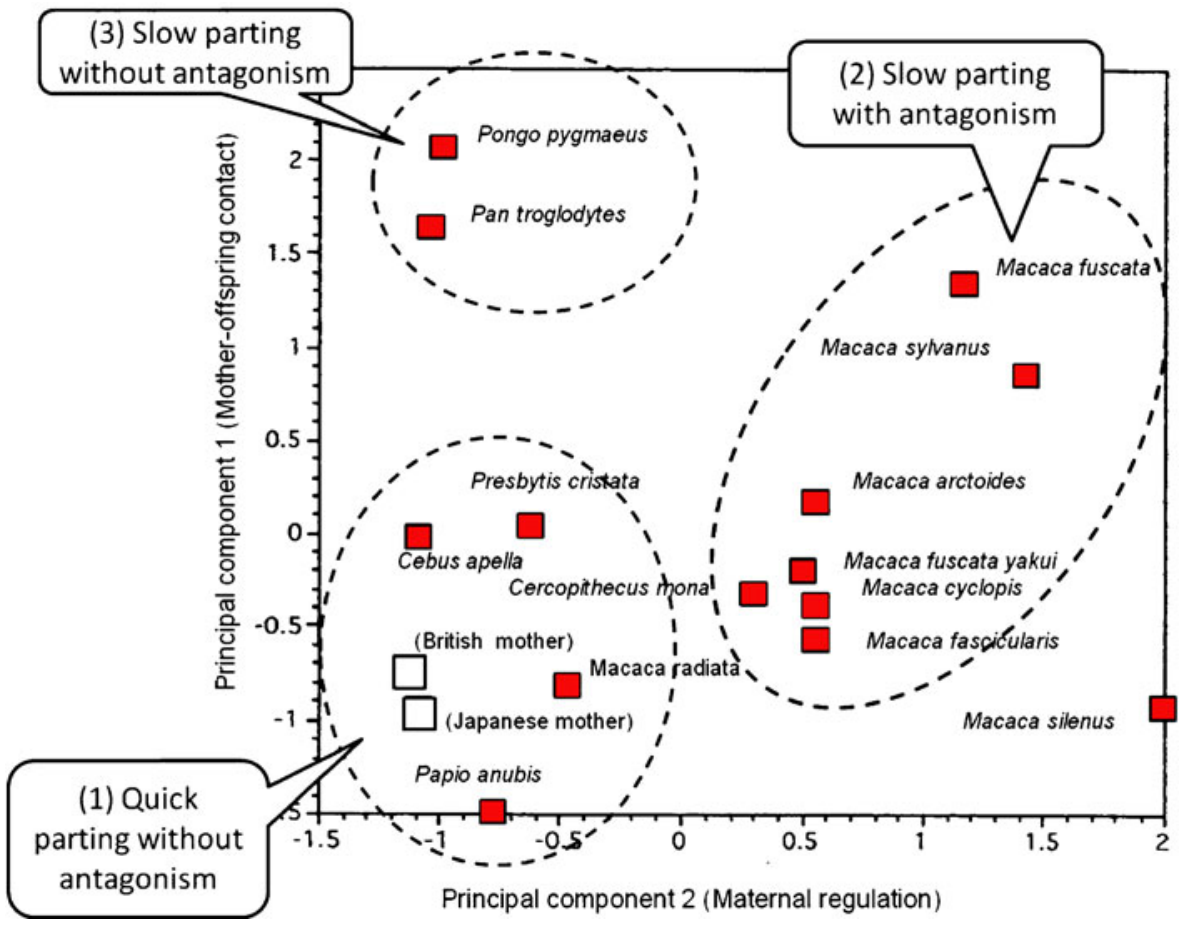

Fig. 1 Principal component analysis of primate mother-offspring relationships (from Neayama 1996) 
initiative in the departure (e.g., bonnet monkey Macaca radiata), (2) slow parting with antagonism in which offspring continue contact and mothers reject it (e.g., Japanese monkey Macaca fuscata), and (3) slow parting without antagonism in which mothers are protective and offspring are also close to the mother for a relatively long time (e.g., chimpanzee Pan troglodytes). The human groups are grouped in the first type, but what actualizes our separation is totally different from the other species. The large distance in the early human mother-offspring dyads is a result of infant behavioral immaturity rather than precocity, actualized with the assistance of surrounding objects, persons and institutions that protect the offspring on behalf of the mothers. The protection by the agents is an extremely important aspect of human Kowakare.

The mother-offspring conflict model has typically been proposed to explain weaning, but a conflict is thought to exist even between fetus and pregnant mother (Haig 1993). An offspring is also not always seeking contact. Not only the mother but also the offspring may act as agents of the negativity in such negative interactions. According to Bateson (1994), mother's negative behavior towards the offspring triggers both dynamic assessment of the offspring's demand by the mother and availability of the mother's resource by the offspring. What is important in Bateson's model is an emphasis on mother-offspring 'cooperation' to promote mutual autonomy rather than a simple antagonism. The idea of adaptive antagonism in Kowakare is in the model.

Negativity is not only a matter of active rejection or aggression between mother and offspring but also of their spontaneous leaving from the partner in a less antagonistic fashion. The separation may be induced by their approach to objects/persons/institutions around them as well as by mother-offspring repulsion. In this sense, mother and offspring are open to the outer systems containing plenty of attractants.

The environment contains both positive and negative resources for any living thing, in which the positive resources are sought and accepted, and the negative resources are avoided, for survival. When offspring are immature and incompetent in handling the resources on their own, their parents assist them. Mammalian mothers play an extremely important role in protecting offspring by pregnancy as well as breastfeeding (Clutton-Brock 1991).

In this respect, mothers are an interface between environment and young offspring that process environmental resources into a form acceptable by the offspring and return the offspring's bodily wastes back to the environment. To do these things is a burden for the mothers, though. Kowakare during the early stage actualizes the reduction of the mothers' burden by gradually withdrawing the role.

\section{Characteristics of Early Human Kowakare}

Bipedalism characterizing human primates caused an immature birth due to a reduction of birth canal diameter and an increase of fetus head size (Jolly 1985). The immaturity at birth, however, does not mean a lack of cognitive competence of human newborn. On the contrary, he or she is born with developed visual cognition 
(Bower 1979) but with immaturity in physical mobility. This resulted in greater need for attention-paying and care-taking of the offspring.

The mother is the primary biological interface between environment and offspring as mentioned above, and the surrounding networks are the secondary socio-cultural interface, enabling the offspring to redirect the target of behavior from mother to other agents. Those two interfaces correspond to the two frameworks of early human Kowakare: the primary (biological) framework and the secondary (socio-cultural) framework. The primary framework is related to inter-body antagonism between mother and offspring, and can be exemplified by feeding/weaning and aversion to bodily products by the offspring as a result of feeding. The secondary framework is the nested systems of objects, persons, and institutions intervening and compensating for the inter-body antagonistic relationship between mother and offspring (Fig. 2).

The secondary framework is largely a unique characteristic of human child care, and is different from culture to culture. Kowakare is a process of decrease in the contribution of mother as the primary interface and increase in the contribution of the secondary interface. Human child care is characterized by its allomothering nature.

\section{Primary Framework}

\section{Feeding and Weaning}

One of the typical bodily resources concerned is nutrients. Breastfeeding is a way for mothers to provide nutrition to the offspring and is a biological interface between environment and offspring. Breast milk is a product of the mothers' own body from what they eat. Mothers try to reject their offspring when the offspring reach a certain level of development, but the offspring are initially reluctant to accept it. This is a cause of weaning conflict proposed by Trivers (1974), and it could promote the offspring's independence. Weaning is thus an important cue to understanding the positive function of maternal negativity.

Intensity and duration of primate mothers' aggression differ from species to species, and humans are characterized by a large mother-infant distance and little aggression as shown above. McDade (2001) discussed supporting and discouraging factors of breastfeeding in human society. A combination of formula milk and bottle is an alternative of breastfeeding. A WHO report (WHO 1981) clearly indicates that there is a huge diversity in human breastfeeding duration, and this is undoubtedly due to the presence of formula. One of the most important reasons for human

Fig. 2 Mother and objects/ persons/institutions as primary and secondary interfaces between environment and offspring. Arrows indicate flows of resources (from Negayama 2006)

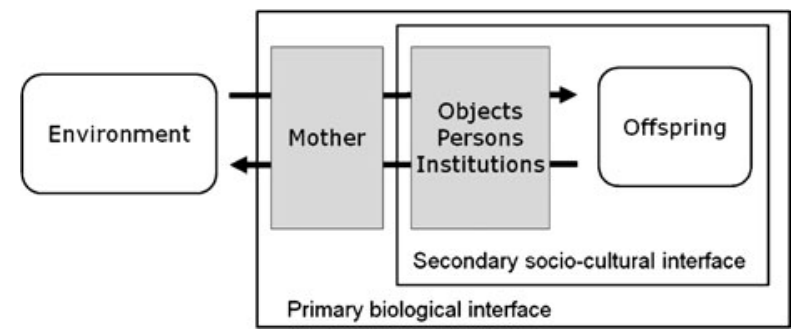


weaning is a perceived insufficiency of breast milk, which may cause conflict between mother and demanding infant. Formula is often introduced following the mother's perception of insufficiency, and it acts as a solution of the conflict, particularly in Japan (Negayama, Norimatsu, Barratt, and Bouville, under submission).

There are various methods of human weaning (Hill and Simpson 1985). Some of which have negative components and others not. The practice of La Leche League mothers is a typical example of non-negative weaning, where the decision on the time to wean is left to the child. On the other hand, 'Dan-nyu' by the Oketani method in Japan (Fig. 3) is an interesting example of weaning with mother's negativity.

The method was originated by a Japanese midwife, Oketani, and is now known all over Japan. She stressed an importance of breastfeeding, and the method of forced stopping was introduced as mothers and children sometimes became too attached to each other and found it difficult to wean at a desired time. The method is basically a sudden stopping of breastfeeding on a certain predetermined day after 1 year of age when the child is already taking solid foods well and walking. Mother secretly draws a cartoon face on her breast immediately after a final feed. Then she shows it when the infant demands the breast the next time and never allows him/her to suckle any more. By this procedure most of the children are successfully weaned within a week, which demonstrates how well mothers' negativity works if it meets with offspring's readiness.

Weaning is essentially a matter of the inter-body nutritional framework between mother and offspring, but it is influenced by many more determinants in humans, such as preparing and providing solid foods. In solid feeding, mother shows an empathetic mouth opening/closing at the moment of the child's food-taking (Negayama 1993), with a peak in frequency at 9 months. The decrease of the behavior reflects a psychological state of mothers underlying weaning as Kowakare. Commercial baby foods are another option for human feeding which reduces the maternal physical burden, although it still is her burden financially. This makes human weaning more complex than in the case of other mammals.

Night-time bed sharing is associated with the continuation of breastfeeding (Pollard et al. 1999). Breastfeeding could cause a conflict with the mothers' relationship with their husband (i.e. the baby's father). He influences breastfeeding (Matich and Sims 1992), and night-time demand for breastfeeding may promote weaning and mother-offspring separation by the child sleeping in a separate room.

Fig. 3 'Dan-nyu' by Oketani method. The child is not allowed to suckle any more after the mother typically draws a cartoon face on her breast (from Negayama 2006)

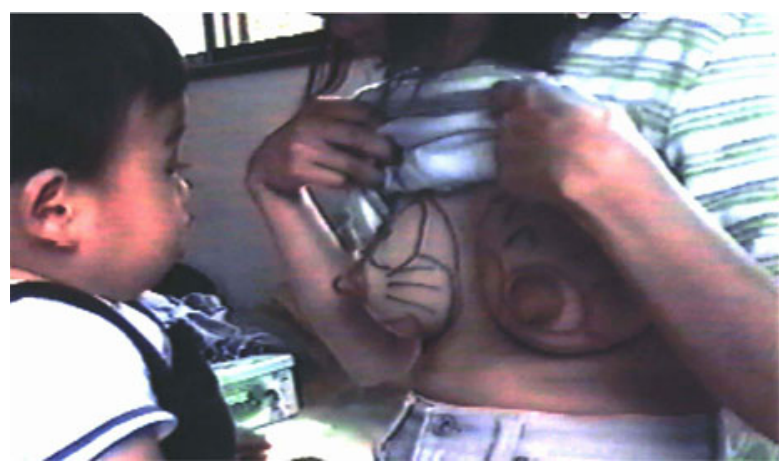




\section{Aversion to Bodily Products of Offspring}

Foods taken by offspring produce various bodily wastes, which is also related with Kowakare. Bodily products, including these wastes, have to be cleaned off the offspring's body in order to keep it sanitary. How parents feel and handle those bodily products of children is another important topic of development in motheroffspring relationship, although the topic has largely been ignored. The bodily products of offspring are mostly aversive to caregivers, but it must be removed in spite of the aversiveness. How parents cope with the aversion could be an interesting question to frame within the process of Kowakare.

Negayama (1998-1999) compared parents' aversion to the bodily products (feces, urine, nose-dirt, pus, phlegm, saliva, etc.) of infants, kindergarten children, and undergraduates. They were asked to estimate how aversive it would be if these things produced by their child, by their own body, or by other persons were in contact with their hand. It was evident that an offspring's bodily products, in spite of their weaker aversiveness compared to those of other persons, were aversive to parents and the aversion increased with age, while aversion to one's own as well as other's products was very similar among the three groups. Above all, feces were most aversive. Negayama (2001) further examined the development of aversion cross-sectionally during the first 4 years, and found that aversion to feces significantly increased between the latter half of the first year and the earlier half of the second year. Other products such as phlegm and grime indicated another significant increase in aversion at around 3 years of age.

Disgust to bodily products of non-kin (alien) persons is biologically adaptive because they may bring germs. Keeping distant from them is a way of avoiding a possible harm to health (Curtis and Biran 2001). This is a reason why the offspring's bodily products are initially less aversive and increases later, and thus this aversion could be a representation of negativity causing Kowakare. However, it should be noted that mothers may develop self-confidence in their child care by managing the negative feeling towards those bodily products from their child.

Secondary Framework: Allomothering with Interface of Objects/Persons/Institutions

Another significant window to understanding Kowakare is the introduction of objects as an interface between mother and offspring. A set of formula plus bottle discussed above is an example, and child-care goods and toys in general are also classified in this category as far as they produce separation between mother and offspring. In this sense, houses could be included in the same category as they provide a safe space to actualize separation between mother and offspring.

Objects also could cause injury to offspring, and hence are ambivalent for mother-offspring dyads. Separation from mother increases the risk of injury in the offspring, which makes mothers cautious and protective. Maternal supervision is an important factor in offspring's accident-proneness (Schwebel et al. 2006), which again is connected to Kowakare because separation is a dilemma. Immediate reactions of mother and child to a big earthquake reveal an interesting developmental trend of the parents' protectiveness and the child's autonomy (Negayama 2010). Parents were surprised by the earthquake, but the younger children showed little or no surprise, and even kept sleeping. Frightened reactions were more evident after 
7 years of age. Parents' typical reaction was to protect the children by embracing them or lying over them at the cost of their own safety, but this decreased with the children's age and grasping of hands increased as children became older.

Another important issue to be addressed is the relation between joint attention and Kowakare. Joint attention is related to the development of intention-reading at about 9 months of age, and important for the infants' cultural learning through taking the mothers' perspective (Tomasello 1993; Tomasello et al. 1993). Normally, joint attention is a matter of triadic relationship between mother, infant and object, and it is related to the above discussion of Kowakare with the presence of objects as a secondary interface.

Infant recognition of communicative intention has been discussed mainly in the domain of visual and auditory modalities (Csibra 2010). However, mother and infant often play together by using their body as the target, and such tactile communication may be an important channel to share intention with others. Tickling is an interesting game as ticklishness can be generated only by the stimulation given by others (Claxton 1975). Initially it is not play, but just a mild stimulation to arouse neonates, but it becomes a cause of joyful reaction of infant by the age of 7 months (Negayama and Yamaguchi 2005). It uses particular body areas as targets of the stimulation, which produces a situation similar to a triadic relationship.

In a normal triadic relationship, in a context of the secondary intersubjectivity (Trevarthen and Hubley 1978), rapid alteration of visual orientation by the infant between target object and mother is a sign of understanding other's intention (BaronCohen 1991; Oyabu 2004). Ishijima and Negayama (unpublished) have conducted a preliminary case study on tickling and ticklishness, and have found a similar alteration of visual orientation between the target body part and the mother's face at about 7 months. The child also showed an expectant ticklishness before the actual touch. The precursor of triadic relationship is an important question to be answered (Rodríguez 2007), and our observation suggests the possibility of 'Proto-triad relationship' (transitional stage from dyad to triad) using the offspring's body as a target at 2 months earlier than the appearance of the true triadic relationship. This proto-triad relationship may act as a precursor of the actual triadic relationship involving an object. It should be confirmed.

Use of certain body areas as the target of tactile stimulation has another important and unique effect, that of automatic sharing of the same emotion, as the bodies of both mother and offspring are expected to be symmetrical with the same sensitivity to contact. Tactile communication is unique in several points. It is always bilateral and simultaneous; the experience is exclusively personal; it can be sent and received at any bodily area; it covers the strongest emotions of love and hate. Thus it should be a source of strong empathy not obtained from interaction using a third external object. We can feel touch on a body area simply by watching another person being touched the same area (Blakemore et al. 2005), and ticklishness also could be shared by a tickling mother and a tickled infant.

Tickling is a behavior with intrusiveness in itself and is a slightly negative tactile stimulus. The joyfulness is enhanced between the mother and offspring by manipulating the negativity: the mother comes to tease the infant by delaying contact for a little while; the infant also stays motionless to solicit tickling from the mother; mother often uses a song and starts tickling at a peripheral body part and 
ends with the ticklish zone of the armpit, for instance. Song is used to share a narrative between mother and infant which enhances their joy by delaying and synchronizing the narrative and its climax (Malloch 1999-2000; Trevarthen 19992000). Thus offspring are not just passively waiting for the mothers' protection but actively seeking for a chance to participate in interaction, and mothers are also not just trying to be responsive to offspring. In such interactions, mother and offspring are in a companionship through dynamically cooperating in the play.

Kowakare is also actualized by persons surrounding mother and offspring. Among other things, the father (e.g., Lewis 1986) and grandparents (Buss 1999) of the offspring are important agents. Care provided by those persons can be summarized under a category of allomothering or allocare. Care sharing is not limited to skin relations, and non-kin specialists such as medical doctor, nursery nurse and teacher (Lamb and Ahnert 2006) are also involved. These persons and their institutions could promote mother-offspring separation, as substitutes for the mother, and could be a situation of Kowakare. Making use of such social systems, e.g., giving birth to children in a hospital, taking a sick child to a clinic, buying formula or commercial baby food at a store, and employing a nanny, are all examples of allomothering through using institutions consisting of object-person systems.

The initial introduction to institutions like day nursery may cause distress reactions from the child immediately after separation. Bronfenbrenner (1979) proposed an ecological approach for child development, and a good relationship between family and those institutions is important for successful Kowakare. Children dynamically move across different situations where objects, persons, and institution are in nested systems, and culture frames the nested systems.

\section{Importance of Kowakare in Early Human Child-care}

Kowakare is a framework to understand the developmental function of negativity in mother-offspring relationship. As discussed above, negativity is seen in normal interactions between human mother and offspring. It has a positive function in promoting autonomy and independence in the offspring, and hence in the mother as well, when it is executed in an optimal combination with positive behaviors.

Negative interaction exists not only on the maternal side but also on the side of the offspring, and crying and resistance to caregivers are typical negative behaviors by the offspring. Crying is more frequent in the home than in day nursery (Negayama et al. 2008), and this is interpreted as non-compliance of offspring to parents in a private context. It is self-assertiveness, particularly common in the Japanese home that works to foster autonomy. Self-regulation is develop more strongly in the public context of day nursery. Children adapt by switching their behavior when moving between the different contexts.

Practices in child-care are different from culture to culture, and the cultural difference should be understood more precisely by evenly considering both the negativity and the positivity. Attachment and Kowakare are not alternatives, they are complementary, and combining the two theories should become more important in the future study of early mother-offspring relationship. 
Maternal behaviors can be placed on the 2-dimensional space with two axes of Positivity-Negativity and Separation-Contact (Fig. 4). Maternal behavior characterized by Positivity and Contact is "care". That has been thought of as the normal maternal behavior. Attachment is a matter of this zone, and mother-offspring interactions have been expected to be within this zone. On the other hand, a zone of Contact and Negativity is typified by "violence", whereas a zone of Separation and Negativity by "neglect". These have been categorized as child abuse and thought to be abnormal. If mother-offspring interactions are constantly in these zones, the relation is viewed as a target for intervention. The 4th zone of Positivity and Separation is for "autonomy", an adaptive separation in human mother-offspring dyads.

Then where is Kowakare located? Human behavioral immaturity in infancy results in normal mother-infant separation, and the separation is actualized by a variety of allomothering agents. In the sense that Kowakare has negative components as has been discussed, it should be in the second or third zone. However, it promotes the offspring's autonomy and that is linked to the fourth zone as exemplified here by weaning, parental aversion to offspring's bodily products, and taking children to institutions. Even positive interactions make offspring avoidant if they are too intense and persistent, as is the case with cut-off behavior or rejection of passive feeding. On the other hand, mildly negative behavior in tickling and peek-a-boo games enhances the positivity.

Thus Kowakare is not able to be limited to any of single zone. The zone should encompass all of the four zones, mixing negativity and positivity. In this zone, positive and negative interactions are dynamically displaced by each other, making the relationship exciting and mutually more autonomous. What is important here is the idea of a dynamic shift and balance between negativity and positivity. Negativity is needed in the healthy development of the mother-offspring relationship.

An optimal level of negativity is sought between mother and offspring: If it is deficient, the relationship becomes boring, and if it is excessive, the offspring withdraws and the equilibrium is regained. This is somehow like what Devinney et al. (2001) described by dynamic assessment. Maternal negativity works to trigger monitoring and assessing the state of the offspring: If it is acceptable, the offspring becomes more autonomous: if it is too harsh, the offspring resists and the mother reduces the negativity. Antagonism is a trigger of negotiation and mutual attunement, and flexible shifting of states is necessary for that to occur.

Mothering based on too much dependency on professionals' advice or the media without their own careful monitoring of the offspring's state would result in rigidity

Fig. 4 Two dimensional representation of maternal behaviors and Zone of Kowakare. (from Negayama 2006)

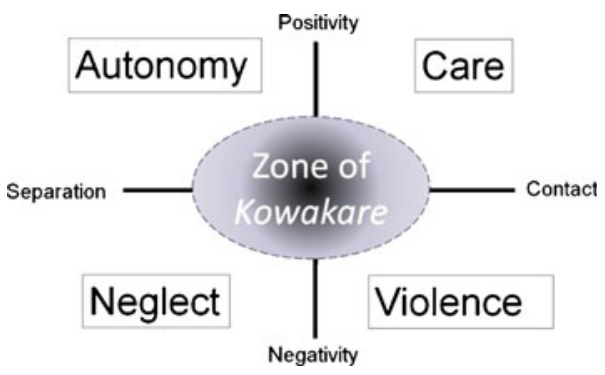


in the child care. Then the flexibility and attunement to the offspring's state is lost and the relationship might become difficult. Too much positivity or overprotection also spoils children, as in the case of adolescent withdrawal.

Figure 5 is a summary of the discussion of this paper. Kowakare is the process of the accumulation of the offspring's 'Otherness' and of mother-offspring mutual autonomy. The otherness in early development has divergent bio-social components (immunological, behavioral, nutritional, and social). The pressent discussion has been focused on the early development of the independence of offspring from mother. Their inter-body antagonism, based on the child's dependence on the mother and on the maternal burdens over nutrition, hygiene, and safety, is the primary framework of human Kowakare. The framework, largely shared with other mammals, is the cause of mother-offspring conflict and rejection.

On the other hand, the secondary framework of objects, persons, and institutions are all interfaces between mother and offspring, compensating the inter-body antagonism, and hence related to allomothering. The framework has a nested structure and is highly developed in humans. It promotes mother-offspring parting by the facilitation of leaving rather than by the active antagonism. Culture influences both the primary and the secondary frameworks, but the latter is much more profoundly determined by it. The two frameworks are connected with each other as, for example, feeding, toileting, and holding are carried out using tools. However, the objects could be a cause of injury, and the accident caused by the objects is a matter of safety, a component of the primary framework.

Inter-body relationship experienced in tactile play such as tickling has another important aspect as a precursor of the mother-offspring-object triad facilitating

Kowakare $=$ The process of accumulation in children's 'Otherness' and
mother-offspring mutual autonomy (Rejection \& leaving)

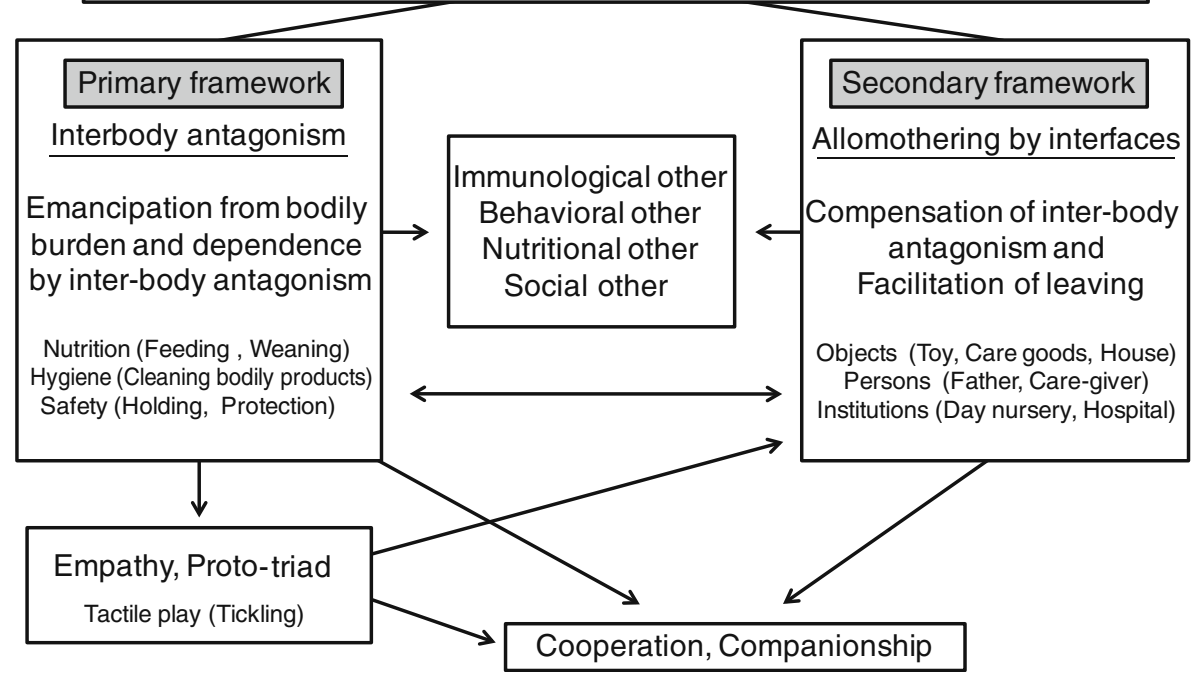

Fig. 5 Schematic representation summarizing discussion of human early Kowakare. Examples are italicized in parentheses 
mother-offspring empathy and the proto-triad relationship. It may incubate the ability of understanding other's intention in the offspring. However, another possibility should be noted, that the ability to understand other's intention enables the child to oppose and manipulate the mother's intentions. This is a basis of negotiation. The mutuality which arises out of that fosters cooperation and companionship between mother and offspring as autonomous individuals.

The present discussion has focussed on early development. Other types of psycho-somatic Kowakare, like conflict over values after the acquisition of language (symbolic other) or sexual antagonism (reproductive other) between parents and offspring, will exist in later development. Reunion and nursing of senescent parents by their grown-up children are further important topics. Those are beyond the scope of the present article and remain to be discussed.

Open Access This article is distributed under the terms of the Creative Commons Attribution Noncommercial License which permits any noncommercial use, distribution, and reproduction in any medium, provided the original author(s) and source are credited.

\section{References}

Baron-Cohen, S. (1991). Precursors to a theory of mind: Understanding attention in others. In A. Whiten (Ed.), Natural theories of mind: Evolution, development, and simulation of everyday mindreading. Oxford: Blackwell.

Bateson, P. (1994). The dynamics of parent-offspring relationships in mammals. TREE, 9, 399-403.

Blakemore, S.-J., Bristow, D., Bird, G., Frish, C., \& Ward, J. (2005). Somatosensory activations during the observation of touch and a case of vision-touch synaesthesia. Brain, 128, 1571-1583.

Bower, T. G. R. (1979). Human development. San Francisco: Freeman.

Bowlby, J. (1969). Attachment and loss: Vol. 1 Attachment. London: Hogarth.

Bronfenbrenner, U. (1979). The ecology of human development: Experiments by nature and design. New York: Harvard University Press.

Buss, D. M. (1999). Evolutionary psychology: The new science of the mind. Boston: Allyn \& Bacon. Claxton, G. (1975). Why can’t we tickle ourselves? Perceptual and Motor Skills, 41, 335-338.

Clutton-Brock, T. H. (1991). The evolution of parental care. Princeton: Princeton University Press.

Csibra, G. (2010). Recognizing communicative intentions in infancy. Mind \& Language, 25, 141-168.

Curtis, V., \& Biran, A. (2001). Dirt, disgust, and disease: Is hygiene in our genes? Perspectives in Biology and Medicine, 44, 17-31.

Devinney, B. J., Berman, C. M., \& Rasmussen, K. L. R. (2001). Changes in yearling rhesus monkeys' relationships with their mothers after sibling birth. American Journal of Primatology, 54, 193-210.

Fairbanks, L. A., \& McGuire, M. T. (1988). Long-term effects of early mothering behavior on responsiveness to the environment in vervet monkeys. Developmental Psychobiology, 21, 711724.

Haig, D. (1993). Genetic conflicts in human pregnancy. The Quarterly Review of Biology, 68, 495-532.

Hansen, E. W. (1966). The development of maternal and infant behavior in the rhesus monkey. Behaviour, 27, 107-149.

Harlow, H. F. (1958). The naure of love. American Psychologist, 13, 673-685.

Hill, V., \& Simpson, M. (Eds.). (1985). Breastfeeding, child health and child spacing: Cross-cultural perspectives. London: Croom Helm.

Jolly, A. (1985). The evolution of primate behavior (2nd ed.). New York: Macmillan.

Lamb, M. E., \& Ahnert, L. (2006). Nonparental child care: Context, concepts, correlates, and concequences. In W. Damonn \& R. M. Lerner (Eds.), Handbook of child psychology, 6th ed. Vol. 4: Child psychology in practice (pp. 950-1016). New Jersey: Wiley.

Lewis, C. (1986). The role of the father in the human family. In W. Sluckin \& M. Herbert (Eds.), Parental behavior (pp. 228-258). Oxford: Basil Blackwell. 
Mahler, M. S., \& Perriere, K. L. (1965). Mother-child interaction during separation-individuation. Psychoanalytic Quarterly, 34, 483-494.

Main, M., \& Stadtman, J. (1981). Infant response to rejection of physical contact by the mother. Journal of American Academy of Child Psychiatry, 20, 292-307.

Malloch, S. N. (1999-2000). Mothers and infants and communicative musicality. Musicae Scientiae, Special Issue, 29-57.

Matich, J. R., \& Sims, L. S. (1992). A comparison of social support variables between women who intend to breast or bottle feed. Social Science \& Medicine, 34, 919-927.

McDade, T. W. (2001). Parent-offspring conflict and the cultural ecology of breast-feeding. Human Nature, 12, 9-25.

Negayama, K. (1981). Maternal aggression to its offspring in Japanese monkeys. Journal of Human Evolution, 10, 523-527.

Negayama, K. (1993). Weaning in Japan: a longitudinal study of mother and child behaviours during milkand solid-feeding. Early Development and Parenting, 2, 29-37.

Negayama, K. (1996). Mother offspring parting in non-human primates and humans. Seishonen-mondai, 43, 26-31 (in Japanese).

Negayama, K. (1998-1999). Development of parental aversion to offspring's bodily products: A new approach to parent-offspring relationships. Annual Report of Research and Clinical Center for Child Development, 22, 51-58.

Negayama, K. (2001). Development of parental aversion toward offspring's body and bodily products. Human Science, 13, 2-13 (in Japanese with English summary).

Negayama, K. (2002). Human mother-infant relationship in comparison with non-human primates: From the perspective of mother-offspring conflict. Japanese Psychological Review, 45, 399-410 (in Japanese with English summary).

Negayama, K. (2006) Child-rearing as Kowakare. Tokyo: Nippon-Hoso-Shuppan-Kyokai (in Japanese).

Negayama, K. (2010). Reactions of children and parents to an earthquake: From the viewpoint of 'Kowakare'(Mutual autonomy). Japanese Journal of Developmental Psychology, 21, 386-395. (in Japanese with English summary).

Negayama, K., \& Yamaguchi, H. (2005). Development of mother-infant tickling play and infant's ticklishness. Journal of Child Health, 64, 451-460.

Negayama, K., Kawahara, N., Fukukawa, S., \& Hoshi, J. (2008). Comparison of toddler's behavior in home and day nursery environments, referring especially to crying. Child, Youth and Environmental Studies, 4, 41-47 (in Japanese with English summary).

Oyabu, Y. (2004). Joint attention: Development from neonates to 2.5-year-olds. Tokyo: Kawashima-shoten. (in Japanese).

Pollard, K., Fleming, P., Young, J., Sawczenko, A., \& Blair, P. (1999). Night-time non-nutritive sucking in infants aged 1 to 5 months: relationship with infant state, breastfeeding, and bed-sharing versus roomsharing. Early Human Development, 56, 185-204.

Rodríguez, C. (2007). Object use, communication, and signs: The triadic basis of early cognitive development. In J. Valsiner \& A. Rosa (Eds.), The Cambridge handbook of sociocultural psychology (pp. 257-276). Cambridge: Cambridge University Press.

Rosenblum, L. A., \& Harlow, H. F. (1963). Approach-avoidance conflict in the mother-surrogate situation. Psychological Reports, 12, 83-85.

Schwebel, D. C., Hodgens, J. B., \& Sterling, S. (2006). How mothers parent their children with behavior disorders: Implications for unintentional injury risk. Journal of Safety Research, 37, 167173.

Simpson, M. J. A., Simpson, A. E., Hooley, J., \& Zunz, M. (1981). Infant-related influences on birth intervals in rhesus monkeys. Nature, 290, 49-51.

Tomasello, M. (1993). On the interpersonal origins of self-concept. In U. Neisser (Ed.), The perceived self: Ecological and interpersonal sources of self-knowledge (pp. 174-184). Cambridge: Cambridge University Press.

Tomasello, M., Kruger, A. C., \& Ratner, H. H. (1993). Cultural learning. Behavioral and Brain Sciences, 16, 495-552.

Trevarthen, C. (1999-2000). Musicality and the intrinsic motive pulse: Evidence from human psychobiology and infant communication. Musicae Scientiae, Special Issue, 155-215.

Trevarthen, C., \& Hubley, P. (1978). Secondary intersubjectivity: Confidence, confiding and acts of meaning in the first year. In A. Lock (Ed.), Action, gesture and symbol: the emergence of language (pp. 183-229). London: Academic.

Trivers, R. L. (1974). Parent-offspring conflict. American Zoologist, 11, 249-264. 
Van der Horst, F. C. P., LeRoy, H. A., \& van der Veer, R. (2008). "When strangers meet": John Bowlby and Harry Harlow on attachment behavior. Integrative Psychological \& Behavioral Science, 42, 370-388.

World Health Organization. (1981). Contemporary patterns of breast-feeding. Geneva: WHO.

Koichi Negayama, born in 1951, is a professor of developmental psychology in the Department of Human Environmental Sciences, Faculty of Human Sciences at Waseda University since 1996. He has a bachelor's degree in psychology and a master's degree in developmental psychology from Osaka University, and is a developmental psychologist with $\mathrm{Ph} . \mathrm{D}$. from Osaka University. He served on the faculty at Osaka University from 1977 to 1986, at Mukogawa Women's University from 1986 to 1996, and at Waseda University from 1996 to the present. Professor Negayama's research is in the area of parentchild interaction with a focus on naturalistic field work. He was originally a primatologist working on mother-offspring aggression. The current research focuses on cultural variety in mother-offspring interbody relationship and Kowakare in Japan and UK, with a special interest in feeding/weaning, holding, injury-proneness, allomothering, etc. 